\title{
Adaptive Fuzzy Sliding Mode Controller for Robot Manipulator: Effect on External Interferences
}

\author{
Sunil C.Kalshetti, S .K. Dixit
}

\begin{abstract}
In the previous decades, the SMC approach has attained unique consideration as this technique offers a systematic model to maintain robust performance and asymptotic stability. As robotic manipulators turn out to be gradually more significant in industrial automation, robotic manipulators by means of SMC have raised as a significant region of research. Hence, this paper intends to model and establish an adaptive sliding mode controller (SMC) for robotic manipulator. As it is not feasible to match up the SMC functions with the system model each time, this paper implements a Fuzzy Inference System (FIS) to replace the system model. It effectively achieves the experimentation in two phases. Accordingly, in the first phase, it attains the accurate features of the system model based on varied samples to characterize the robotic manipulator. Consequently, it derives the obtained features as fuzzy rules. In the subsequent phase, it signifies the derived fuzzy rules depending on adaptive fuzzy membership functions. Moreover, it establishes the self-adaptiveness using Grey Wolf Optimization (GWO) to attain the adaptive fuzzy membership functions. The analysis distinguishes the efficiency of the adopted technique with the optimal investigational scheme and the traditional schemes such as SMC, Fuzzy SMC (FSMC) and GWO-SMC. Moreover, the comparative analysis is also performed by including the external disturbances and noise and validates the effectiveness of the proposed and conventional models.
\end{abstract}

Keywords: Sliding Mode Control; Robot manipulators; Controller; Load variation, External Disturbances

Revised Manuscript Received on November 06, 2019.

\begin{tabular}{|c|c|}
\multicolumn{2}{c}{ Nomenclature } \\
\hline Abbreviation & Description \\
\hline SMC & Sliding Mode Controller \\
\hline FIS & Fuzzy Inference System \\
\hline GWO & Grey Wolf Optimization \\
\hline SAGWO & Self-Adaptive GWO \\
\hline FSMC & Fuzzy Logic Controller \\
\hline FLC & Adaptive Fuzzy SMC \\
\hline AFSMC & Two Degrees Of Freedom \\
\hline 2DOF & Artificial Neuro-Fuzzy Inference System \\
\hline ANFIS & Proportional Integral Derivative \\
\hline PID & Second Order Terminal SMC \\
\hline PSO & Second Order Smc \\
\hline SOTSM & Positive Big \\
\hline SOSMC & Positive Medium \\
\hline PB & Positive Small \\
\hline PM & Zero \\
\hline PS & Negative Big \\
\hline Z & Negative Medium \\
\hline NB & Negative Small \\
\hline NM & \\
\hline NS & \\
\hline & \\
\hline
\end{tabular}

\section{INTRODUCTION}

Robotics [1] [2] is a comprehensively examined topic owing to its appropriateness in appliances such as in bio-hazardous regions such as toxic places, nuclear plants and moreover in high accurate tasks such as microsurgery, laser cutting, etc. The majority of the robot manipulators [3] [4] with reduced cost chiefly include servo motors that consist of an interior controller. The control techniques are modeled to endure certain factors that affect the system when attaining excellent tracking performance. Such techniques chiefly resort to an increased controller gain for lessening the uncertainty of the system. It is known that robotic manipulators should meet with the uncertainties namely, disturbance, load variation and friction occurring in the system. In addition, it is much complex to accomplish better performance while the control design is entirely dependent on the robotic plant design [5] [6]. Numerous techniques were tracked to deal with this issue such as model predictive control, feedback linearization, and SMC [7] [8]. SMC exists as an enhanced control technique that was extensively deployed due to its easiness in modeling, reliable performance and order diminution features [9]. It is the effective robust control method that offers invariance characteristics to the entire system since the system arrives at the reaching point of SMC [10] [11]. 
SMC has recognized itself as an effectual control method that was confirmed to be robust in opposition to external disturbances and system uncertainties. The SMC method [12] is modeled for driving the system state constraints to equilibrium by exploiting a discontinuous feedback control theory. This approach encloses constructive invariance features in terms of irregularities in the plant representation and therefore, it is an excellent aspirant for controlling uncertain nonlinear systems. In recent times, FSMC was also established, that was proven to be an excellent device for controlling the undefined systems and variants of parameters [13]. On articulating the necessary characteristics of heuristic engineering, an FLC can deal with up harsh with healthy irregularities by merging it with SMC. However, SMC [14] [15] includes limited appliances in the areas of robotics owing to its high frequency and thus it is found to be unsuitable for realistic applications. Hence, analyzation is carried out to minimize SMC chattering for formulating it appropriate for robotics appliances.

The major contribution of this paper is depicted below.

1. This paper intends to present a new adaptive SMC for robotic manipulator using FIS model under the condition of external disturbances and noises.

2. In the first phase of presented work, accurate features of the system model are attained based on varied samples to characterize the robotic manipulator and thus fuzzy rules are extracted.

3. Here, in the second phase, it signifies the derived fuzzy rules depending on adaptive fuzzy membership functions. Moreover, it establishes the SA-GWO approach to attain the adaptive fuzzy membership functions.

4. Finally, the performance of adopted SA-GWO method is compared over other traditional schemes to validate the effectiveness of the adopted method.

The overall organization of the work is as follows: Section II portrays the literature work. Section III describes the proposed adaptive fuzzy-based SMC controller for PUMA 560 robot. Section IV discusses experimental outcomes, and Section V concludes the paper.

\section{LITERATURE REVIEW}

Nabanita et al. [1] have introduced the scheme for executing dynamic controller on a manipulator that does not include drive joints. The adopted controller exploits a non-singular sliding surface for attaining time constancy in addition to superior tracking behavior. In order to evade a structurally multifaceted control theory and the flexible nonlinearities of the manipulator were evaluated by means of the time delay concept, in which deferred signals were exploited to approximate the nonlinearities of the model. The whole system was confirmed by means of effective analytical, and simulation analysis.

In 2018, Abbas et al. [2] have established the hybrid position control of robotic manipulators functioning in tentative surroundings by incorporating the FLC with traditional SMC. The established AFSMC necessitates the least particulars regarding the dynamic structure of manipulator and physical features between the other hybrid control techniques offered until now. In reality, it does not require assessment of the dynamic model and uncertainties in advance. At last, simulation outcomes demonstrated the superior performance of the established controller in managing with improbabilities.

In 2017, Vijay and Jena [3] have suggested the controlling approach of 2DOF robot manipulator depending on the coupling of ANFIS with SMC. For controlling the robot manipulator, SMC with PID sliding surface was introduced. The constraints of the sliding surface were attained by reducing performance indices by means of PSO. At last, an ANFIS controller was implemented, and it was established to be extremely robust regarding the instabilities in input torque.

In 2014, Mondal and Chitra [4] have introduced an adaptive SOTSM for regulating the robotic manipulators. In the adopted controller, the time derivative was deployed rather than the usual control input. Moreover, an adaptive tuning technique was exploited to handle the uncertainties of the system in which the upper limits were not necessary to be identified previously. The performance of the introduced control approach was assessed by the control of a 2-link robot manipulator. Finally, the investigational outcomes revealed the efficiency of the adopted control scheme.

In 2017, Kali et al. [5] have examined the issues of joint space trajectory tracking regarding accuracy. A discretetime SOSMC merged with time delay evaluation was modeled to formulate the joint positions in tracking a trajectory with improved accurateness. Furthermore, satisfactory evaluation guarantying the convergence of error to zero was offered. Experimentations were also obtainable to substantiate the improved performance of the adopted controller.

\section{PROPOSED ADAPTIVE FUZZY-BASED SMC CONTROLLER FOR PUMA 560 ROBOT}

\section{A. Robotic Model}

The modeling of the dynamics of a serial n-link robot is specified in Eq. (1) in which $\dot{u}$ denotes the joint velocities in $n \times 1$ vector, $u$ denotes the joint displacements in $n \times 1$ vector, $\tau$ point outs torque of actuators, in $n \times 1$ vector, $M(u) \quad$ signifies symmetric positive inertia matrix in $n \times n$ vector, $c(u, \dot{u})$ points out the torques of Coriolis and centripetal in $n \times 1$ vector and $g(u)$ denotes the gravitational torque in $n \times 1$ vector. In addition, owing to gravity $g(u)$ is produced as the potential energy gradient $U(u)$.

$$
M(u) \ddot{u}+c(u, \dot{u})+g(u)=\tau
$$

Assume that the joints of robot are correlated along with the revolute joints. Let $u_{d}$ be the necessary joint positions, $u_{d}$ is considered as the function of the double differentiable vector. The actuator torque is approximated by introducing the control issu so that Eq. (2) is accomplished that promotes the suitable control aim.

$$
\lim _{t \rightarrow \infty} u(t)=u_{d}(t)
$$

The present simulation regards the "DOF PUMA-560 robot", with the set up of six joints. Moreover depending on [18], 
he dynamical and kinematical features of the arm are introduced. The PUMA motors are offered with commercially relevant DC motors. As a result, the evaluation concerning the size and power of PUMA motors provides the electrical constraints of the motors.

\section{B. Proposed System Model}

The structural design of the controlling method depending on the adaptive fuzzy system is revealed in Fig. 1. The adopted approach is introduced to fine-tune the joint angles of the "PUMA 560 robot arm". Accordingly, the actual feedback is produced from the real "PUMA 560 system" that is linked to the corresponding control law initiator. In addition, the required actual feedback and trajectory are exploited to calculate the differential error function $(D E)$ and error function $(E)$. The sliding surface generator produces the activating signal depending on the evaluated $E$. In the meantime, the sliding mode variables are tuned by the adopted adaptive fuzzy system using an improved meta-heuristic SAGWO scheme that can further generate the joint angle with minimized error as in the predetermined format.

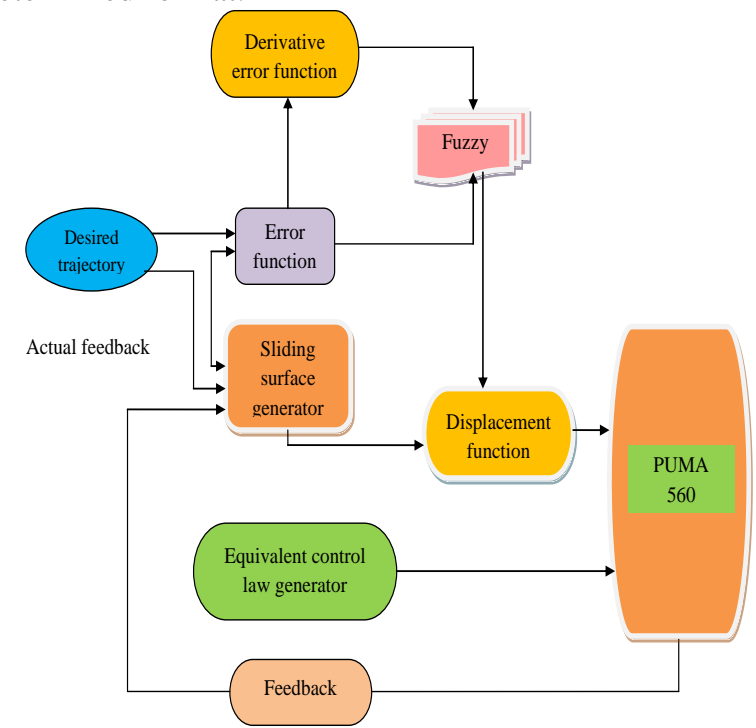

Fig 1. Overall framework of the proposed control scheme

Two inputs namely, $E$ and $D E$ are applied to the fuzzy system. According to the confines of the specified inputs, they are allocated as PB, PM, PS, Z, NB, NM, and NS. The limits of Z, PM, PS, NM, and NS are dependent on the triangular membership function and the limits NB and PB are dependent on trapezoidal membership function. The fuzzy system produces the related rules with the aforementioned input confines that are regarded as the constants of sliding mode. Thus, the produced sliding mode variables are entirely dependent on exploited $E$ and $D E$. Here, Table 1 portrays the sliding mode variables or rules produced by the fuzzy system.

Table 1: SMC constants or rules produced by fuzzy system

\begin{tabular}{|c|c|c|c|c|c|}
\hline E/D & $\mathrm{ENB}$ & NM|NS Z & PS & & \\
\hline NB & NB & NB $\mid$ NB & $\mathrm{NM}$ & & \\
\hline NM & NB & \begin{tabular}{l|l}
$\mathrm{NB}$ & $\mathrm{NM}$ \\
\end{tabular} & INS & $Z$ & PS \\
\hline NS & NB & $\mathrm{NMNS}$ & $\mathrm{Z}$ & & PM \\
\hline Z & NB & NMNS Z & PS & & PB \\
\hline S & $\mathrm{NM}$ & \begin{tabular}{l|l}
$\mathrm{NS}$ & $\mathrm{Z}$ \\
\end{tabular} & PN & & \\
\hline
\end{tabular}

\section{\begin{tabular}{|l|l|l|l|l|l|l|l|}
\hline PM & NS & $Z$ & PS & PM & PB & PB & PB \\
\hline
\end{tabular}}

\begin{tabular}{|l|l|l|l|l|l|l|l|}
\hline PB & $Z$ & PS & PM & PB & PB & PB & PB \\
\hline
\end{tabular}

he triangular membership function is denoted by Eq. (4), in which $r$ and $s$ indicate the lower limit and upper limit, $t$ denotes certain value and $x$ refers to the optimal constraint, in which $r<t<s$. Similarly, the demonstration of the trapezoidal membership function is revealed in Eq. (3), in which $v$ and $u$ denote the upper and lower support limit, in which $r<u<v<s$. The limits of membership function for SMC is revealed in Table 2.

$$
\begin{aligned}
& \mu_{1}(x)=\left\{\begin{array}{cl}
0, & x \leq r \\
\frac{x-r}{t-r}, & r<x \leq t \\
\frac{s-x}{s-t}, & t<x<s \\
0, & x \geq s
\end{array}\right. \\
& \mu_{2}(x)=\left\{\begin{array}{cc}
0, & (x<r) \operatorname{or}(x>s) \\
\frac{x-r}{u-r}, & r \leq x \leq u \\
1, & u \leq x \leq v \\
\frac{s-x}{s-v}, & v \leq x \leq s
\end{array}\right.
\end{aligned}
$$

Table 2: Bounds of membership function for SMC

\begin{tabular}{|l|l|l|l|l|l|}
\hline $\mathrm{X}$ & $\mathrm{r}$ & $\mathrm{t}$ & $\mathrm{s}$ & $\mathrm{u}$ & $\mathrm{v}$ \\
\hline $\mathrm{NM}$ & $\mathrm{NB}_{1}$ & $\left(\mathrm{NB}_{2}-\mathrm{NB}_{1}\right) / 2$ & $\mathrm{NB}_{2}$ & - & - \\
\hline $\mathrm{NS}$ & $\mathrm{NM}_{1}\left(\mathrm{NM}_{2}-\mathrm{NM}_{1}\right) / 2$ & $\mathrm{NM}_{2}$ & - & - \\
\hline $\mathrm{PS}$ & $\mathrm{PM}_{1}$ & $\left(\mathrm{PM}_{2}-\mathrm{PM}_{1}\right) / 2$ & $\mathrm{PM}_{2}$ & - & - \\
\hline $\mathrm{PM}$ & $\mathrm{PB}_{1}$ & $\left(\mathrm{~PB}_{2}-\mathrm{PB}_{1}\right) / 2$ & $\mathrm{~PB}_{2}$ & - & - \\
\hline $\mathrm{NB}$ & - & - & - & $\mathrm{NB}_{1}$ & $\mathrm{NM}_{2}$ \\
\hline $\mathrm{PB}$ & & - & - & $\mathrm{PM}_{2}$ & $\mathrm{~PB}_{1}$ \\
\hline
\end{tabular}

\section{Adaptive Membership Function using Grey Wolf Optimization}

Fundamentally, the membership function is portrayed as a "curve that defines how each point in the input space is mapped to a membership value between 0 and 1". The output of fuzzy produces a certain membership function with the produced rules that creates more error according to the traditional fuzzy dependent SMC [16]. As a result, SAGWO model is suggested here to vary the membership function adaptively that have to reduce the error among the desired and actual value.

GWO algorithm [17] is a recent meta- heuristic algorithm that operates depending on the principle behind the hunting characteristics of grey wolves, for catching the prey. Accordingly, three wolves namely $\alpha, \beta$ and $\delta$ holds the role of hunting the corresponding preys. The pattern of hunting is dependent on 3 phases that comprise of (a) tracking, (b) following and (c) catching the prey. On considering the diverse wolves, $\alpha$ is allocated as the leader of the entire wolves that takes the decision concerning sleeping, resting time and hunting of wolves. In addition, the second and third level wolf $\beta$ and $\delta$ aids the leader to take the required decisions. On the other hand, a wolf termed $\omega$ is allowed only for eating. Fig. 2 illustrates the solution encoding constraints of SAGWO model, in which $J$ denotes the collective solution vector to the GWO. 


\section{Adaptive Fuzzy Sliding Mode Controller for Robot Manipulator: Effect on External Interferences}

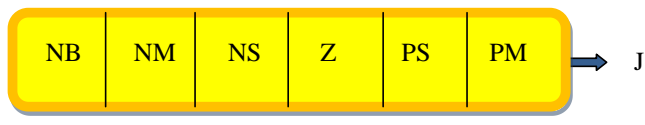

Fig 2. Solution encoding

Therefore, after integrating the fuzzy system, the membership function with GWO scheme is together indicated as in Eq. (5).

$$
\mu=\mu(J)
$$

The objective function of implemented SAGWO dependent SMC in "PUMA 560 robotic arm" is denoted in Eq. (6), i.e. the error among the desired and actual joint angles. In Eq. (6), $\theta_{i}^{A}$ refers to the actual joint angle and $\theta_{i}^{D}$ denotes the desired joint angle. The formulation of the optimal joint angle is revealed in Eq. (7), in which $\varepsilon$ refers to the control waveforms from SMC and $K^{f u z z y}$ signifies the constant portrayed by fuzzy system. Accordingly, the optimal joint angle is determined on the basis of the control signals that are generated from SMC. The evaluation of the control waveform is portrayed in Eq. (8), in which $\varepsilon_{s w}$ and $\varepsilon_{e q}$ denotes the continuous and equivalent part of SMC, correspondingly, denoted in Eq. (9) and Eq. (10) and $s_{i}$ denotes the switching boundary.

$$
\begin{aligned}
& \left.E\right|_{t=t^{\max }}=\sum_{i=1}^{3}\left|\theta_{i}^{D}(t)-\theta^{A}(t)\right| \\
& \theta^{D}=\varepsilon\left(K^{f u z z y}\right) \\
& \varepsilon=\varepsilon_{e q}+\varepsilon_{s w} \\
& \varepsilon_{s w}=-K^{f u z z y} \operatorname{satf}\left(x_{i}\right) \\
& \operatorname{satf}\left(x_{i}\right)=\left\{\begin{array}{lll}
+1, & \text { if } & f\left(x_{i}\right)>s_{i} \\
\frac{f_{i}}{s_{i}} & \text { if } & f\left(x_{i}\right) \leq s_{i} \\
-1, \text { if } & f\left(x_{i}\right)<s_{i}
\end{array}\right.
\end{aligned}
$$

Therefore, the control waveform from SMC is produced dependent on the fuzzy rules as given in Table 1 that further evaluates the optimal joint angle. Further, it adjusts the portrayed joint angle adaptively by the SAGWO model. The encircling pattern of grey wolves is indicated in Eq. (11) in which $C$ and $H$ indicates the coefficient vectors, $J_{p}$ refers to the position vector of prey, $J$ refers to the grey wolves position, and $t$ refers to the present iteration. In addition, the modeling of the vectors $C$ and $H$ are denoted in Eq. (13) and Eq. (14) in which ${ }^{a}$ denotes an element, that is reduced from 2 to 0 and ${ }^{r_{1}} r_{2}$ indicates the arbitrary vectors, which are distributed uniformly among $[0,1]$.

$$
\begin{aligned}
& K=\left|H \cdot J_{p}(t)-J(t)\right| \\
& J(t+1)=J_{p}(t)-C \cdot K \\
& C=2 a \cdot r_{1}-a \\
& H=2 \cdot r_{2}
\end{aligned}
$$

Furthermore, the adopted SAGWO model adapts the value of $a$ dependent on the variation in function. The SAGWO scheme frames the element $a$ as in Eq. (15), in which $\tau$ indicates the variation in fitness functions. The illustration of $\tau$ is indicated in Eq. (16) in which $f(t-1)$ denotes the preceding iteration and $f(t)$ denotes the present iteration.

$$
\begin{aligned}
a & =\left(2-2 \times \frac{1}{\text { Maximum iteration }}\right) \times \tau \\
\tau & =\frac{f(t-1)-f(t)}{f(t-1)}
\end{aligned}
$$

Therefore the hunting characteristics of grey wolves are attained from Eq. (17) to Eq. (22) that offers the relevant position to every wolf. Eventually, the Eq. (23) portrays the update position depending on the position of whole wolves.

$$
\begin{gathered}
K_{\alpha}=\left|C_{1} \cdot J_{\alpha}-J\right| \\
K_{\beta}=\left|C_{2} \cdot J_{\beta}-J\right| \\
K_{\delta}=\left|C_{3} \cdot J_{\delta}-J\right| \\
J_{1}=J_{\alpha}-C_{1} \cdot\left(K_{\alpha}\right) \\
J_{2}=J_{\beta}-C_{2} \cdot\left(K_{\beta}\right) \\
J_{3}=J_{\delta}-C_{3} \cdot\left(K_{\delta}\right) \\
J(t+1)=\frac{J_{1}+J_{2}+J_{3}}{3}
\end{gathered}
$$

\section{RESULTS AND DISCUSSIONS}

\section{A. Experimental Procedure}

The proposed SAGWO-FSMC scheme that adopts the fuzzy model to support the SMC to control the robotic manipulator was simulated in MATLAB, and the outcomes were attained. The count of iteration was fixed as 100. For analyzing the performance of the proposed model, it was distinguished with the traditional experimental schemes such as SMC [1], FSMC [19], and GWO-SMC [17] in case of external disturbances and noises. The basic Simulink model of the SAGWO-FSMC was shown in Fig. 3 and the SAGWO block was broadly modeled in Fig. 4. 


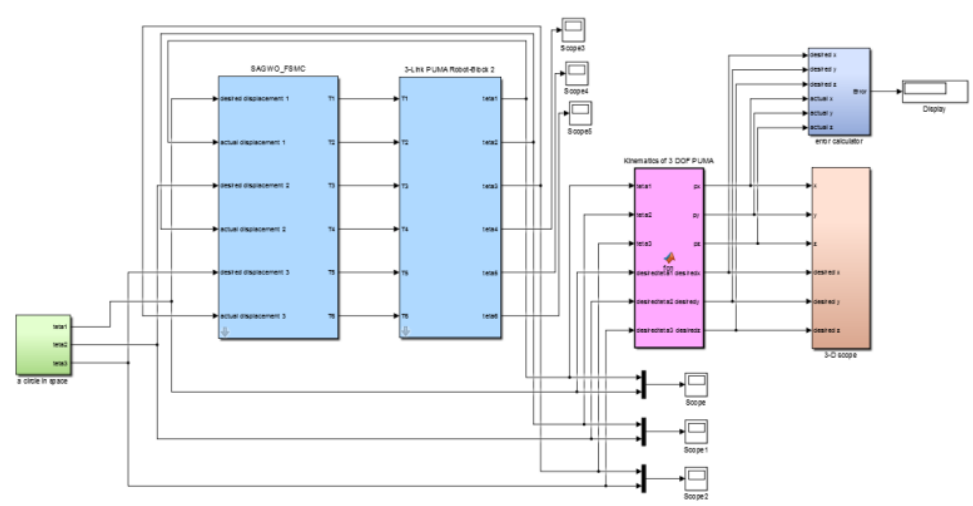

Fig 3. Simulink model of SAGWO-FSMC

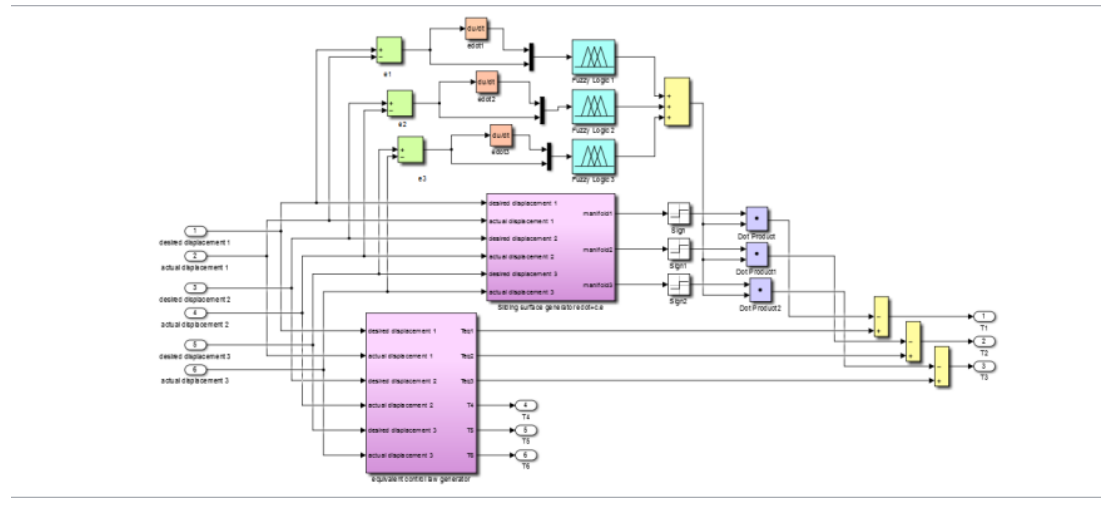

Fig 4. Simulink model of SAGWO block

\section{B. Analysis on Angles}

The time period is fixed at $10 \mathrm{~ms}$, and the mobility of joint angles to be controlled were noted. When distinguishing the actual joint angle $\theta_{1}$ with the optimal $\theta_{1}$ as revealed in Fig. 5 (a), at $2.5 \mathrm{~ms}$, the performance of SMC is $0.02 \%$, FSMC and GWO-FSMC is $0.013 \%$ SAGWO-FSMC is $0.01 \%$, deviated from the desired angle $\theta_{1}$. Therefore, SAGWOFSMC scheme is found be similar to the desired scheme than the traditional GWO-FSMC in controlling $\theta_{1}$.

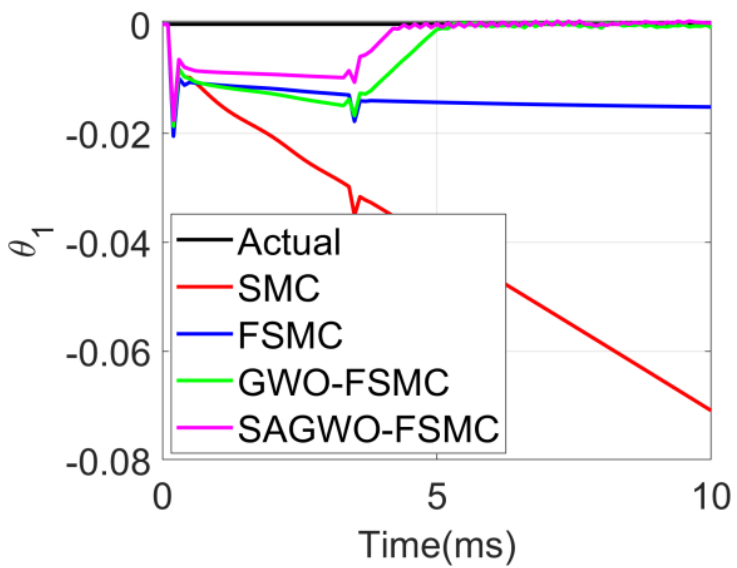

(a)
Similarly, from the analysis on the joint angle $\theta_{2}$ as specified in Fig. 5(b), at $3.5 \mathrm{~ms}$, the actual $\theta_{2}$ of SAGWOFSMC is $2.98 \%$ deviated from the optimal $\theta_{2}$ that is improved than the other schemes. In addition, the actual SAGWO-FSMC at $10 \mathrm{~ms}$ is $1.35 \%$ varied from the optimal $\theta_{3}$, as specified in Fig. 5(c). Thus, the adopted SAGWO-FSMC controls the joint angles, which reveals a high correlation with the optimal joint angles, and hence it shows the development over the traditional SMC models.

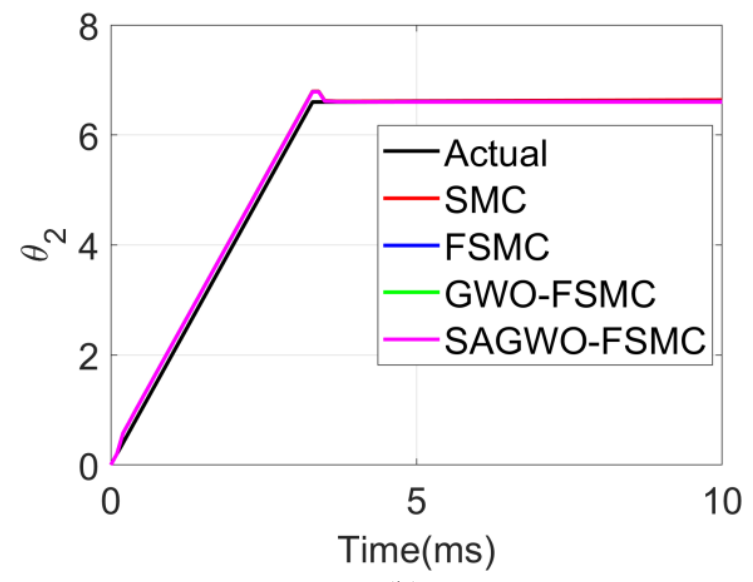

(b) 


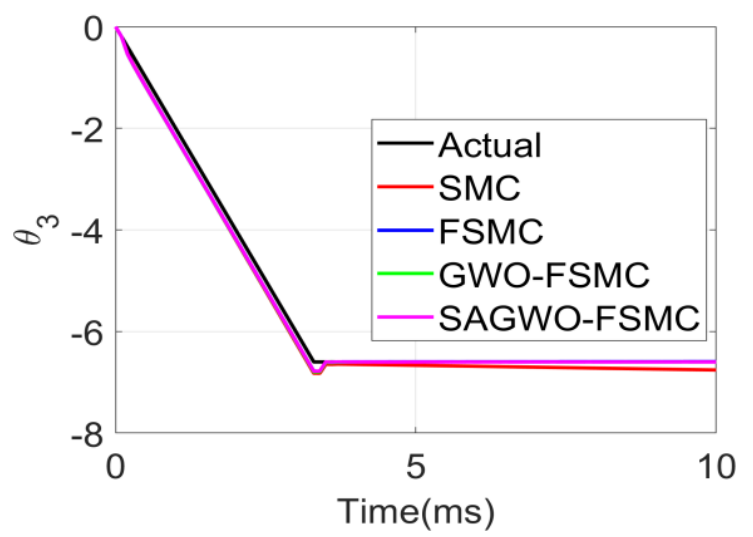

(c)

Fig 5. Performance analysis on three angles of joints with respect to time namely, (a) ${ }^{\theta_{1}}$ (b) $\theta_{2}$ and (c) $\theta_{3}$

\section{Impact of External Disturbance}

The graphical demonstration of the examination on three displacements namely, $x$ y $z$ with time when applying external disturbance, is revealed in Fig. 6. From Fig. 6(a), at $10 \mathrm{~ms}$, the actual value of displacement $x$ is 0.23 , while the SMC has attained a displacement of 0.4, FSMC has attained a displacement of 0.3 , and GWO-FSMC has attained a displacement of 0.3 and the proposed SAGWO-FSMC has attained a displacement of 0.29. Likewise, from Fig. 6(b), at $2 \mathrm{~ms}$, the actual value of displacement $y$ is 0 , while the SMC has attained a displacement of 0.01, FSMC has attained a displacement of 0.005 , and GWO-FSMC has

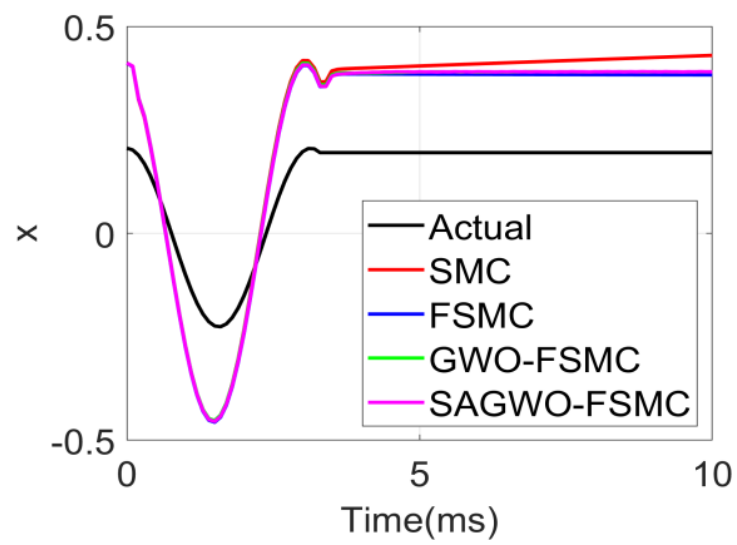

(a) attained a displacement of 0.005 and the proposed SAGWOFSMC has attained a displacement of 0.004. In addition, from Fig. 6(c), at $2 \mathrm{~ms}$, actual value of displacement $z$ is 0 , while the SMC has attained a displacement of 0.01, FSMC has attained a displacement of 0.005 , and GWO-FSMC has attained a displacement of 0.005 and the proposed SAGWOFSMC has attained a displacement of 0.004 . Therefore, the proposed SAGWO-FSMC is found to be adjacent to the actual value, thus showing the superiority of the presented scheme.

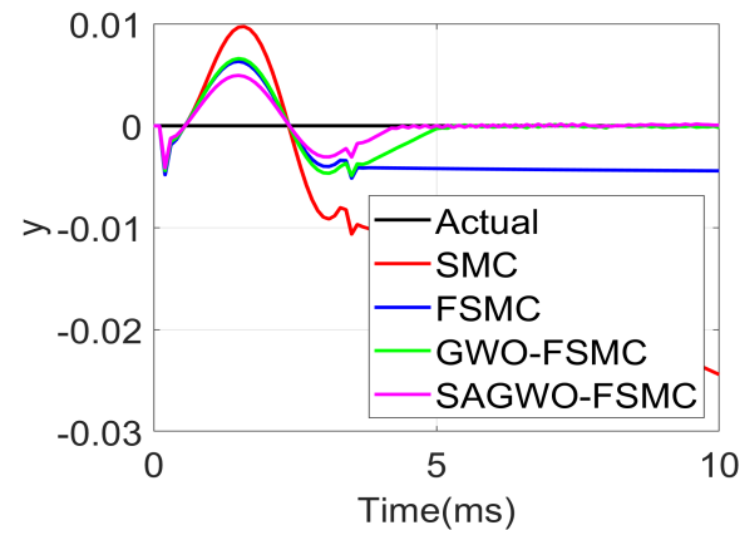

(b)

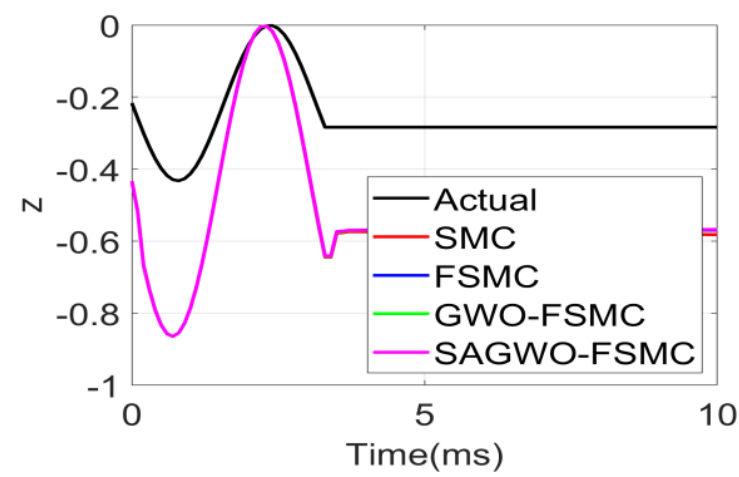

(c)

Fig 6. Impact of external disturbance on three displacements namely, (a) ${ }^{x}$ (b) ${ }^{y}$ and (c) $z$ 


\section{Impact of Noise}

The graphical interpretation of three displacements $x y z$ with respect to time by the impact of noise is revealed by Fig.7. From Fig. 7(a), at $10 \mathrm{~ms}$, the actual value of displacement is 0.4 and the proposed SAGWO-FSMC has attained a displacement of 0.3. Similarly, from Fig. 7(b), at $2 \mathrm{~ms}$, the actual value of displacement is of 0 , the SMC has attained a displacement of $-4 \times 10^{-4}$, FSMC has attained a displacement of $-4 \times 10^{-4}$, and GWO-FSMC has attained a

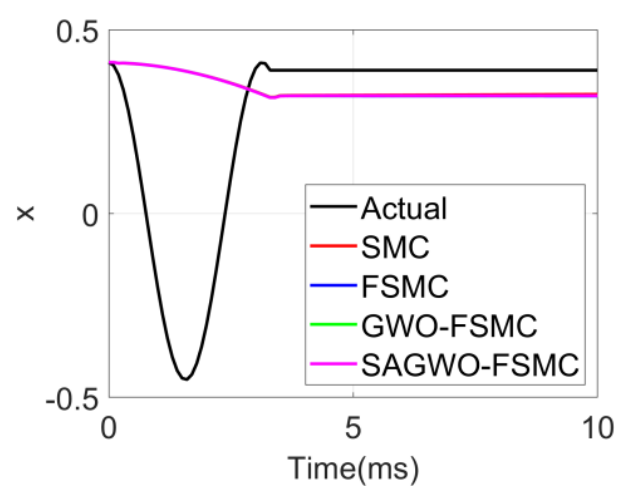

(a) displacement of $-4 \times 10^{-4}$ and the proposed SAGWO-FSMC has attained a displacement of $-3 \times 10^{-4}$. Moreover, from Fig. $7(\mathrm{c})$, at $0 \mathrm{~ms}$, actual value of displacement is of -0.43 and the proposed SAGWO-FSMC has attained a displacement of 0.43 . Therefore, the proposed model is found nearer to the actual displacement in the presence of noise, thus showing the enhancement of the presented model.

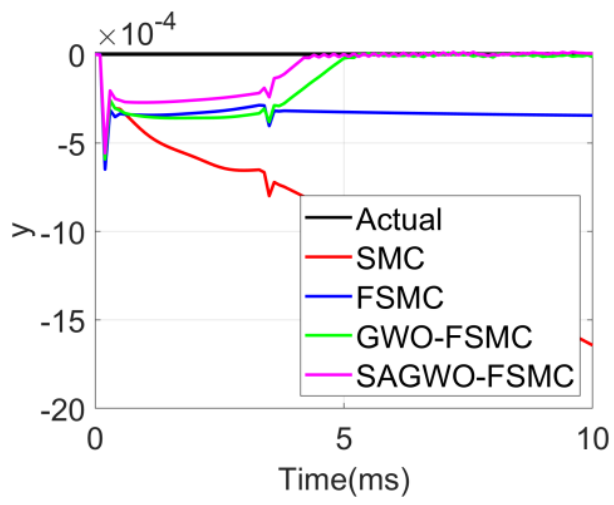

(b)

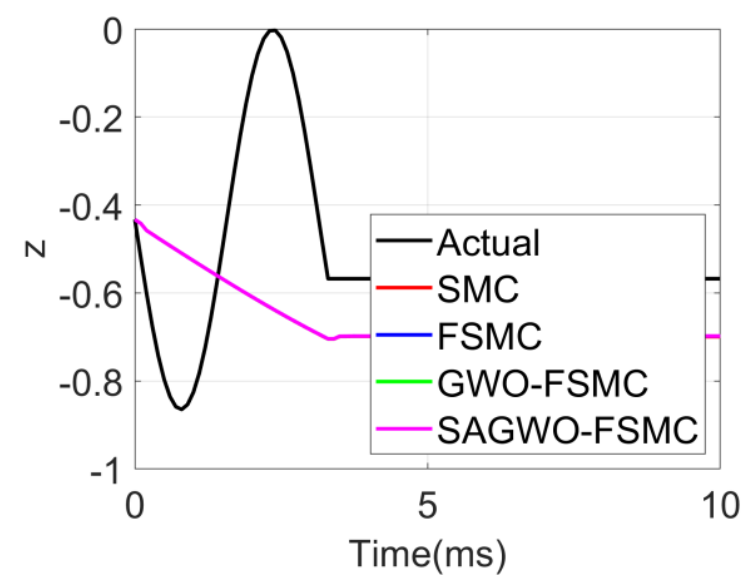

(c)

Fig 7. Impact of noise on three displacements namely, (a) ${ }^{x}$ (b) ${ }^{y}$ and (c) $z$

\section{E. Computational Time}

The analysis on computational time for the proposed SAGWO-FSMC-based controller for the robotic manipulator is given by Table 3 . From the analysis, when the time is $0 \mathrm{~ms}$, the adopted SAGWO-FSMC scheme is $5.31 \%$ better than SMC, $0.41 \%$ better than FSMC and $0.39 \%$ better than GWO-FSMC approaches. In addition, when the time is $3 \mathrm{~ms}$, the implemented SAGWO-FSMC method is $3.32 \%$ superior to SMC, $0.37 \%$ superior to FSMC and $1.55 \%$ superior to GWO-FSMC algorithms. On considering the time period of $10 \mathrm{~ms}$, the adopted SAGWOFSMC model is $10.08 \%$ better than SMC, $0.74 \%$ better than FSMC and $0.79 \%$ better than GWO-FSMC techniques. Thus, the enhanced performance of the presented SAGWOFSMC scheme in maintaining better computational time has been proven.

Table 3: Analysis on computational time

\begin{tabular}{|r|r|r|r|r|}
\hline Time & SMC [1] & $\begin{array}{c}\text { FSMC } \\
{[19]}\end{array}$ & $\begin{array}{c}\text { GWO- } \\
\text { FSMC [17] }\end{array}$ & $\begin{array}{c}\text { SAGWO- } \\
\text { FSMC }\end{array}$ \\
\hline 0 & 0.33947 & 0.32275 & 0.32269 & 0.32143 \\
\hline 1 & 0.4114 & 0.41088 & 0.41006 & 0.41169 \\
\hline 2 & 0.083813 & 0.089249 & 0.085669 & 0.091279 \\
\hline 3 & 0.32461 & 0.31499 & 0.31877 & 0.31384 \\
\hline 4 & 0.35503 & 0.34445 & 0.34458 & 0.34307 \\
\hline 5 & 0.35938 & 0.34471 & 0.34458 & 0.34283 \\
\hline 6 & 0.36369 & 0.34469 & 0.34427 & 0.34263 \\
\hline 7 & 0.36794 & 0.34468 & 0.34473 & 0.34245 \\
\hline 8 & 0.37214 & 0.34465 & 0.34473 & 0.34229 \\
\hline 9 & 0.37628 & 0.34464 & 0.34477 & 0.34216 \\
\hline 10 & 0.38037 & 0.34458 & 0.34479 & 0.34204 \\
\hline
\end{tabular}

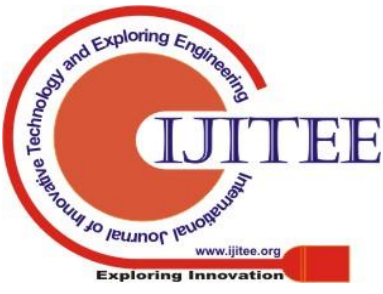




\section{Adaptive Fuzzy Sliding Mode Controller for Robot Manipulator: Effect on External Interferences}

\section{CONCLUSION}

This paper has introduced the improved performance of robotic manipulator namely "PUMA 560 robotic arm". Here, adaptive SMC was executed for the robotic manipulator on concerning the external disturbances and noises. Generally, a system representation was not feasible to merge with the functioning of SMC each time. Therefore, FIS was deployed in this research work to substitute the system model. Accordingly, the experimentation was performed depending on two phases. The accurate features from the system model beneath a variety of samples were attained in the initial phase to indicate the robotic manipulators, while the attained features were allocated as fuzzy rules. In contrast, adaptive fuzzy membership function was exploited to portray the attained fuzzy rules in the subsequent phase, by means of the SAGWO scheme. Finally, the valuable comparative analysis was done by validating the performance of proposed over conventional models while adding external disturbances and noise in the manipulator.

\section{REFERENCES}

1. Nabanita Adhikary, Chitralekha Mahanta, "Sliding mode control of position commanded robot manipulators", Control Engineering Practice, vol. 81, pp. 183-198, December 2018.

2. Abbas Karamali Ravandi, Esmaeel Khanmirza, Kamran Daneshjou, "Hybrid force/position control of robotic arms manipulating in uncertain environments based on adaptive fuzzy sliding mode control", Applied Soft Computing, vol. 70, pp. 864-874, September 2018.

3. M. Vijay, Debashisha Jena, "PSO based neuro fuzzy sliding mode control for a robot manipulator", Journal of Electrical Systems and Information Technology, vol. 4, pp. 243-256, no. 1, May 2017.

4. Sanjoy Mondal, Chitralekha Mahanta, "Adaptive second order terminal sliding mode controller for robotic manipulators", Journal of the Franklin Institute, vol. 351, no. 4, pp. 2356-2377, April 2014.

5. Yassine Kali, Maarouf Saad, Khalid Benjelloun, Abdelilah Fatemi, "Discrete-time second order sliding mode with time delay control for uncertain robot manipulators", Robotics and Autonomous Systems, vol. 94, pp. 53-60, August 2017.

6. Mohd Salim Qureshi, Pankaj Swarnkar, Sushma Gupta, "A supervisory on-line tuned fuzzy logic based sliding mode control for robotics: An application to surgical robots", Robotics and Autonomous Systems, vol. 109, pp. 68-85, November 2018

7. Luis Gracia, J. Ernesto Solanes, Pau Muñoz-Benavent, Alicia Esparza, Josep Tornero, "Cooperative transport tasks with robots using adaptive non-conventional sliding mode control", Control Engineering Practice, vol. 78, pp. 35-55, September 2018.

8. M. Vijay, Debashisha Jena, "Backstepping terminal sliding mode control of robot manipulator using radial basis functional neural networks", Computers \& Electrical Engineering, vol. 67, pp. 690-707, April 2018.

9. Manjusha Bhave, S. Janardhanan, L. Dewan, "Configuration Control of Planar Underactuated Robotic Manipulator using Terminal Sliding Mode", IFAC-PapersOnLine, vol. 49, no. 1, pp. 148-153, 2016.

10. J. Ernesto Solanes, Luis Gracia, Pau Muñoz-Benavent, Jaime Valls Miro, Josep Tornero, "Human-robot cooperation for robust surface treatment using non-conventional sliding mode control", ISA Transactions, vol. 80, pp. 528-541, September 2018.

11. Zhiqiang Ma, Guanghui Sun, "Dual terminal sliding mode control design for rigid robotic manipulator", Journal of the Franklin Institute, 4 February 2017.

12. Minh-Duc Tran, Hee-Jun Kang, "Adaptive terminal sliding mode control of uncertain robotic manipulators based on local approximation of a dynamic system", Neurocomputing, vol. 228, pp. 231-240, 8 March 2017.

13. Mehran Rahmani, Ahmad Ghanbari, Mir Mohammad Ettefagh, "Hybrid neural network fraction integral terminal sliding mode control of an Inchworm robot manipulator", Mechanical Systems and Signal Processing, vol. 80, pp. 117-136, 1 December 2016.
14. Magdy M. Abdelhameed, "Enhancement of sliding mode controller by fuzzy logic with application to robotic manipulators", Mechatronics, vol. 15 , no. 4, pp. 439-458, May 2005

15. Seong Ik Han, Jangmyung Lee, "Finite-time sliding surface constrained control for a robot manipulator with an unknown deadzone and disturbance", ISA Transactions, vol. 65, pp. 307-318, November 2016.

16. Srinivasan Alavandar and M.J. Nigam, "Fuzzy PD + I control of a six DOF robot manipulator" Industrial Robot: An International Journal, vol. 35, no.2, pp. 125-132, 2008.

17. Seyedali Mirjalili, Seyed Mohammad Mirjalili, and Andrew Lewis, "Grey Wolf Optimizer" Advances in Engineering Software, vol. 69, pp. 46-61, March 2014.

18. B. Armstrong, O. Khatib, and J. Burdick, "The explicit dynamic model and inertial parameters of the PUMA 560 arm," Proceedings. 1986 IEEE International Conference on Robotics and Automation, pp. 510518, 1986.

19. Ruey-Jing Lian, "Enhanced adaptive grey-prediction self-organizing fuzzy sliding-mode controller for robotic systems", Information Sciences, vol. 236, pp. 186-204, 1 July, 2013. 